\title{
Modulation of Ki67 and myogenic regulatory factor expression by tocotrienol-rich fraction ameliorates myogenic program of senescent human myoblasts
}

\author{
Chun Min Tan, Nadwa Aqeela Mohd Najib, Nur Farahin Suhaimi, Nur Alia Halid, Vi Vien Cho, \\ Saiful Idham Abdullah, Muhammad Zulhilmi Ismail, Shy Cian Khor, Faizul Jaafar, Suzana Makpol
} Department of Biochemistry, Faculty of Medicine, Universiti Kebangsaan Malaysia,
Kuala Lumpur, Malaysia

Submitted: 4 May 2018; Accepted: 8 September 2018; Online publication: 3 June 2019

Arch Med Sci 2021; 17 (3): 752-763

DOI: https://doi.org/10.5114/aoms.2019.85449

Copyright (๑) 2019 Termedia \& Banach

\section{Abstract}

Introduction: Replicative senescence results in dysregulation of cell proliferation and differentiation, which plays a role in the regenerative defects observed during age-related muscle atrophy. Vitamin $\mathrm{E}$ is a well-known antioxidant, which potentially ameliorates a wide range of age-related manifestations. The aim of this study was to determine the effects of tocotrienol-rich fraction (TRF) in modulating the expression of proliferation- and differentiation-associated proteins in senescent human myoblasts during the differentiation phase.

Material and methods: Human skeletal muscle myoblasts were cultured until senescence. Young and senescent cells were treated with TRF for $24 \mathrm{~h}$ before and after differentiation induction, followed by evaluation of cellular morphology and efficiency of differentiation. Expression of cell proliferation marker Ki67 protein and myogenic regulatory factors MyoD and myogenin were determined.

Results: Our findings showed that treatment with TRF significantly improved the morphology of senescent myoblasts. Promotion of differentiation was observed in young and senescent myoblasts with TRF treatment as shown by the increased fusion index and larger size of myotubes. Increased Ki67 and myogenin expression with TRF treatment was also observed in senescent myoblasts, suggesting amelioration of the myogenic program by TRF during replicative senescence.

Conclusions: TRF modulates the expression of regulatory factors related to proliferation and differentiation in senescent human myoblasts and could be beneficial for ameliorating the regenerative defects during aging.

Key words: tocotrienols, replicative senescence, myoblasts, myotubes, differentiation.

\section{Introduction}

Skeletal muscle regeneration is the process that is activated for repair of damaged or injured muscle fibers in response to muscle cell necrosis, eventually maintaining muscle structure and function. The regenerative capacity of skeletal muscle is disrupted with aging, resulting in regenerative failure and development of sarcopenia, age-related muscle atrophy [1]. Satellite cells are a small cell population located between the basal lamina and sarcolemma of myofibers, which have an important role in

\author{
Corresponding author: \\ Suzana Makpol \\ Department of Biochemistry \\ Faculty of Medicine \\ Universiti Kebangsaan \\ Malaysia \\ Kuala Lumpur, Malaysia \\ Phone: +603-91459554 \\ E-mail: suzanamakpol@ \\ ppukm.ukm.edu.my
}


muscle regeneration [2]. With appropriate stimuli, satellite cells can escape from the quiescent state and initiate proliferation to expand the cell population [3]. The proliferating satellite cells are called myoblasts. Subsequently, myoblasts will differentiate and fuse to form new myofibers or repair the existing injured fibers [4]. But, the interplay of intrinsic and extrinsic age-related alterations in satellite cells has contributed to the regenerative defects during aging [5-11].

Both extensive proliferation and terminal differentiation processes are highly coordinated, with several molecules being activated in sequence to signal the myoblasts to accomplish muscle regeneration [4]. However, similar to other human somatic cells, myoblasts have a limited proliferative life span, in which the cells will ultimately reach the irreversible growth arrest, known as replicative senescence [12]. This limited proliferative capacity could be the intrinsic change of satellite cells that accounts for the regenerative defects during aging. A study showed that geriatric satellite cells tend to convert into senescence (geroconversion) under proliferative pressure, which promotes cellular senescence and affects normal regenerative function [11]. Sustained suppression of senescence pathways could be imperative to preserve satellite cells and prevent diminishing regenerative capacity with aging $[11,13]$.

Replicative senescence affects myogenic differentiation in myoblasts resulting in the production of impaired myotubes [14]. The expression of the factors regulating myogenic differentiation, such as myogenic regulatory factors (MRFs), is deregulated in senescent myoblasts $[15,16]$. The MRFs, which include Myf5, MyoD, myogenin and MRF4, are the key transcription factors in the myogenic specification, differentiation and maintenance [17]. Not only in senescent myoblasts - a decrease of myogenin expression has also been observed in hindlimb suspension of aged rats, indicating that aging might restrict the increase of MRFs in response to regenerative stimuli [18].

Age-related muscle atrophy can be attributed to the accumulation of reactive oxygen species (ROS) with aging [19]. Introducing an antioxidant could be a potential solution to delay sarcopenia progression. Vitamin $\mathrm{E}$ is a well-known antioxidant, which potentially ameliorates a wide range of age-related manifestations [20]. Previous studies reported that vitamin E deficiency can accelerate aging progression and alter muscle structure and function [21, 22]. Conversely, introducing vitamin E may help to protect against oxidative stress and reduce muscle damage $[23,24]$. Previous research on vitamin $E$ mainly focused on the antioxidant effect of a single form of vitamin E, which is $\alpha$-tocopherol. However, vitamin $E$ consists of two isomers, which are tocopherols and tocotrienols, and each isomer can be further classified into four forms $\alpha-, \beta-, \gamma$ - and $\delta$-. Between these two isomers, tocotrienols are the less-explored isomer of vitamin $\mathrm{E}$.

Tocotrienol isomer has been found to possess greater antioxidant activity as compared to tocopherol [25]. Interestingly, a mixture of vitamin E, particularly tocotrienol-rich fraction (TRF), showed a greater effect in reducing the oxidant level as compared to the single form of vitamin E [26]. Previously, the therapeutic effect of TRF was shown in a stress-induced premature senescence (SIPS) model of myoblasts [27]. Besides its antioxidant activity, vitamin E, particularly TRF, has shown non-antioxidant properties, acting as signaling molecules and modulating gene and protein expression. Moreover, TRF can ameliorate the replicative senescence-related abnormalities, including improving proliferation capacity of senescent myoblasts and promoting the myogenic differentiation via modulation of MRF gene expression [15]. However, our previous study focused on the effect of TRF which was given to myoblast cells during the proliferation phase before stimulation of the differentiation phase. Thus, it would be of interest to determine the effects of TRF in modulating the proliferation- and differentiation-associated protein expression in senescent human myoblasts during the differentiation phase.

\section{Material and methods}

\section{Cell culture and replicative senescence}

Human skeletal muscle myoblasts (HSMM) were purchased from Lonza (Walkersville, MD, USA). The cells were cultured in Skeletal Muscle Basal Medium (SkBM) supplemented with human epidermal growth factor, fetal bovine serum, dexamethasone, L-glutamine and gentamicin/amphotericin B (Lonza, Walkersville, MD USA). Cells were cultivated at $37^{\circ} \mathrm{C}$ in a humid atmosphere containing $5 \% \mathrm{CO}_{2}$. After that, the myoblasts were expanded extensively to reach replicative senescence, which was manifested by the decline in proliferative capacity and accumulation of senescence biomarker in culture [15]. For each passage, the population doubling (PD) of cells was calculated as: $\ln (N / n) / \ln 2$, where $N$ is the number of cells at harvest stage and $n$ is the number of cells at seeding stage. In this study, the cells were divided into 4 groups, which were young control (PD < 15; without treatment), young TRF (PD < 15; with TRF treatment), senescent control ( $P D>20$; without treatment) and senescent TRF ( $P D>$ 20; with TRF treatment).

\section{TRF preparation and treatment protocol}

TRF Gold Tri E 70 (Sime Darby Sdn. Bhd., Selangor, Malaysia) of palm oil that consists of $24.15 \%$ tocopherols and $75.85 \%$ tocotrienols was used as 
the TRF treatment in this study. A stock solution of TRF was freshly prepared in $100 \%$ ethanol $(1: 1)$ and kept at $-20^{\circ} \mathrm{C}$ for not more than 1 month. TRF was then incubated overnight with fetal bovine serum (FBS) at $37^{\circ} \mathrm{C}$ prior to use. TRF was diluted with culture medium (either proliferation medium or differentiation medium) to a final concentration of $50 \mu \mathrm{g} / \mathrm{ml}$ and used to treat the cells for $24 \mathrm{~h}$. The final concentration of TRF used in the present study was taken from our previous study on series of dose-dependent effect reported by Khor et al. [15]. Treatment with TRF for $24 \mathrm{~h}$ at a concentration of $50 \mu \mathrm{g} / \mathrm{ml}$ showed the greatest percentage of viable cells in young and senescent myoblast cells. Furthermore, the myoblast cells used in the present study were similar to the previous study. In this study, the cells were treated twice on separate occasions, which were before the differentiation induction and at day 1 of differentiation.

\section{Induction of myogenic differentiation}

After $24 \mathrm{~h}$ of TRF treatment, the cells were considered at day 0 of differentiation. Proliferation medium SkBM was substituted with DMEM:F12 (Lonza, Walkersville, MD USA) supplemented with $2 \%$ horse serum (ATCC, Baltimore, USA) to induce differentiation. The cells were then treated with TRF in differentiation medium at day 1 of differentiation for $24 \mathrm{~h}$. The induction of myogenic differentiation was continued until day 5 of differentiation and the culture medium was changed every 2 days.

\section{Determination of myogenic purity and analysis of cellular morphology}

By using a mouse monoclonal anti-Desmin antibody (D33; Dako, Produktionvej, Denmark), the myogenic purity and cellular morphology were determined. Desmin is a myogenic lineage specific protein that will not be expressed in fibroblasts. The cells were first fixed in cold ethanol for $5 \mathrm{~min}$. After the blocking step, the cells were sequentially incubated with anti-desmin antibody (1:50) and Alexa Fluor 488 goat anti-mouse (Molecular Probes, Eugene, OR, USA). Nuclei were then visualized using Hoechst 33342 (Molecular Probes, Eugene, OR, USA). The cells were observed under an EVOS FL Digital Inverted Fluorescence Microscope (Thermo Fisher Scientific, USA). In order to determine the myogenic purity, at least 300 cells were counted in three independent cultures to obtain the percentage of desmin-positive cells. At the same time, the morphological changes of myoblasts were observed.

\section{Determination of myogenic differentiation efficiency}

Myotubes were labeled with anti-desmin antibody at day 1 , day 3 and day 5 of differentiation according to the protocol described before [15]. In order to evaluate the efficiency of differentiation, fusion index and myotube size were calculated. The myotube size was estimated based on the number of nuclei per myotube. To determine the fusion index, the formula below was used, and at least 300 nuclei were counted in more than 10 different randomly chosen fields.

$$
\text { Fusion index }=\frac{\begin{array}{c}
\text { The number of nuclei } \\
\text { in myotubes }(>2 \text { nuclei })
\end{array}}{\begin{array}{c}
\text { The total number of nuclei } \\
\text { in desmin positive cells }
\end{array}} \times 100 \% \text {. }
$$

\section{Determination of protein expression}

Protein expression was determined using the immunocytochemistry method. The proliferation marker Ki67 was quantitated at day 0, day 1, day 3 and day 5 of differentiation using anti-Ki67 antibody (sc-23900; Santa Cruz, California) (1 : 50 dilution), while the differentiation-related factors MyoD and myogenin were quantitated at day 1 , day 3 and day 5 of differentiation using the following primary antibodies at the respective dilution: anti-MyoD antibody (5.8A; Novus Biologicals, Canada) (1:100 dilution) and anti-myogenin antibody (F5D; Dako, Produktionsvej, Denmark) (1: 20 dilution). In brief, the cells were fixed with cold ethanol and blocked for the nonspecific binding site before incubating with the corresponding primary antibody at $4^{\circ} \mathrm{C}$ for a different incubation period in accordance with the respective antibody. The incubation period for anti-Ki67 antibody was $1 \mathrm{~h}$ and overnight for both anti-MyoD and anti-myogenin. Alexa Fluor 488 was used as the secondary antibody and Hoechst 33342 was used to visualize the nuclei. Stained cells were observed under an EVOS FL Digital Inverted Fluorescence Microscope (Thermo Fisher Scientific, USA). In order to estimate the protein expression, at least 250 cells were counted in three independent cultures to acquire the percentage of Ki67-positive cells, the percentage of MyoD-positive cells and the percentage of myogenin-positive cells. For Ki67 expression, the percentage was normalized to myogenic purity.

\section{Statistical analysis}

Statistical analyses were performed using SPSS 22.0 software (IBM, NY, USA). Oneway ANOVA was used for multiple comparisons. Post hoc LSD test or Tukey's test was used if equal variance was assumed whereas Dunnett's T3 was selected if non-equal variance was assumed. Data were presented as mean \pm standard deviation (SD) for at least three replications. The result was considered as statistically significant if the $p$ value was less than 0.05 . 
Table I. Myogenic purity of myoblasts

\begin{tabular}{|lcccc|}
\hline Parameter & Young control & TRF-treated young & Senescent control & TRF-treated senescent \\
\hline $\begin{array}{l}\text { Percentage of desmin } \\
\text { positive cells }\end{array}$ & $79.41 \pm 4.94$ & $82.61 \pm 4.18$ & $45.44 \pm 5.10^{\mathrm{a}, \mathrm{b}}$ & $49.90 \pm 4.04^{\mathrm{a}, \mathrm{b}}$ \\
\hline
\end{tabular}

${ }^{a} p<0.05$, significantly different compared to young control; ${ }^{b} p<0.05$, significantly different compared to TRF-treated young cells.

\section{Results}

\section{Myogenicity and cellular morphology} of myoblasts

To elucidate the effect of TRF treatment on replicative senescence, we cultured myoblasts until replicative senescence was reached. Myogenicity and cell morphology were observed in all groups using anti-desmin antibody. Senescent control and TRF-treated senescent myoblasts had a significantly lower myogenic purity compared to both untreated young control and TRF-treated young myoblasts $(p<0.05$; Table I). However, there was no significant difference between the control and TRF-treated group in both young and senescent myoblasts.

Myoblasts were spindle shaped and lacked striation (microfilament) when young (Figures $1 \mathrm{~A}, \mathrm{~B}$ ), but became flatter and larger, with the presence of prominent striations in cytoplasm during replicative senescence (Figure $1 \mathrm{C}$ ). However, TRF-treated senescent myoblasts resembled the young myoblasts, as manifested by the increased number of spindle shaped cells in the culture (Figure $1 \mathrm{D}$ ).
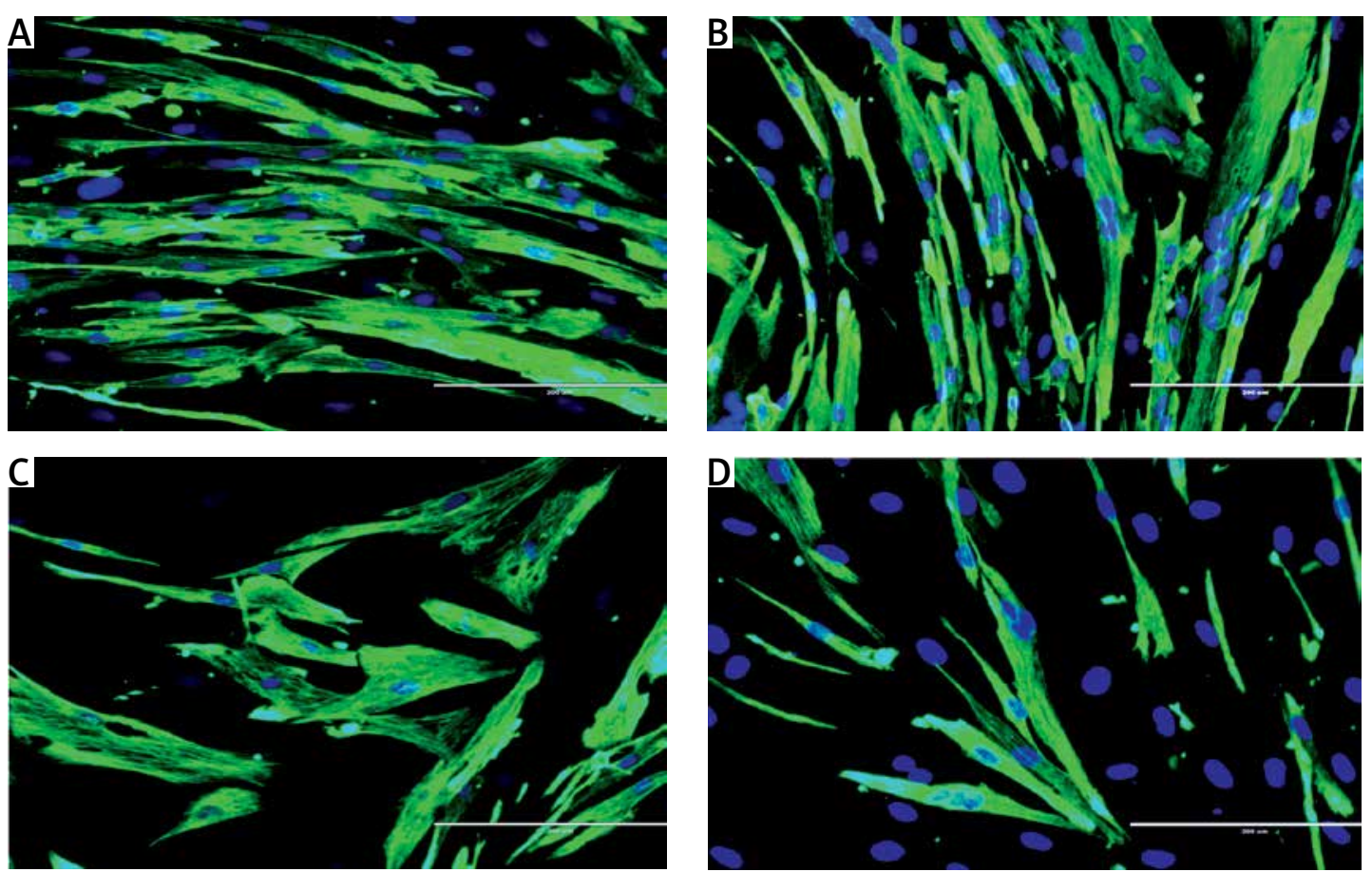

Figure 1. Effects of replicative senescence and TRF treatment on the cellular morphology of myoblasts. The micrographs of myoblasts were captured from A - young control, B - TRF-treated young, C - senescent control, and D - TRF-treated senescent (magnification: 200x, scale bar: $200 \mu \mathrm{m}$ ). Myoblasts were displayed were stained for desmin (green) and Hoechst (blue). Regardless TRF treatment, young myoblasts were displayed in spindle shape. Meanwhile, untreated senescent control cells manifested broader and flatter cytoplasm with prominent striations. On contrary, TRF-treated senescent myoblasts exhibited more spindle shaped cytoplasm which resembled the morphology of young myoblasts 

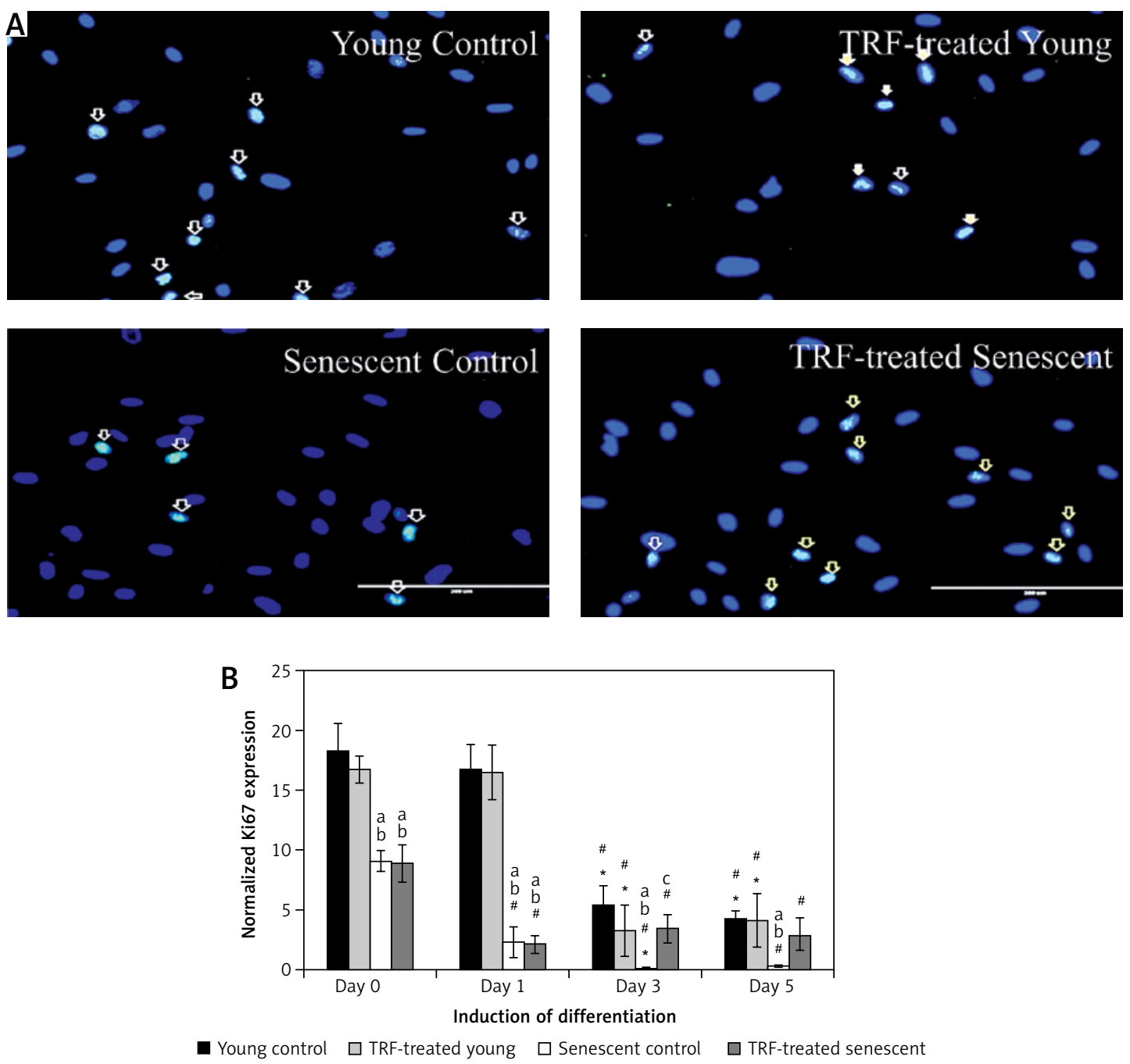

Figure 2. Effects of replicative senescence and TRF treatment on the expression of proliferation marker Ki67. A - Myoblasts were labelled with anti-Ki67 antibody (green) and Hoechst (blue) to detect the presence of Ki67 protein, as indicated by white arrow (magnification 200x, scale bar: $200 \mu \mathrm{m}$ ). B - Quantitative analysis of Ki67 expression in myoblasts at day 0 , day 1 , day 3 and day 5 of differentiation

${ }^{a} p<0.05$, significantly different compared to young control at corresponding day of differentiation; ${ }^{b} p<0.05$, significantly different compared to TRF-treated young myoblasts at corresponding day of differentiation; ${ }^{c} p<0.05$, significantly different compared to senescent control at corresponding day of differentiation; ${ }^{*} p<0.05$, significantly different compared to the corresponding group at day 0 of differentiation; and ${ }^{*} p<0.05$, significantly different compared to the corresponding group at the day 1 of differentiation. Data are presented as the mean $\pm S D, n=3$.

until day 3 of differentiation for control senescent myoblasts $(p<0.05)$ and was maintained for TRF-treated senescent myoblasts. At day 5 of differentiation, the Ki67 protein expression in senescent myoblasts was significantly lower compared to the expression detected on day 0 of differentiation $(p<0.05)$. The Ki67 protein was significantly increased in TRF-treated senescent myoblasts at day 3 of differentiation as compared to the senescent control $(p<0.05)$.

\section{Expression of myogenic regulatory factors MyoD and myogenin}

The modulation of MRF expression by TRF during replicative senescence of myoblasts was investigated by determining the expression of MyoD and myogenin from day 1 to day 5 of differentiation using anti-MyoD or anti-myogenin antibody with Alexa Fluor 488 (green) and Hoechst (blue) (Figures 3 A, 4 A). Our results showed that MyoD expression was significantly increased in TRF-treated young myoblasts at day 1 and day 3 of differentiation compared to control young myoblasts $(p<0.05)$ (Figure 3 B). However, the expression of MyoD in TRF-treated young myoblasts was significantly decreased at day 5 of differentiation compared to day 1 and day 3 of differentiation $(p<0.05)$. In senescent myoblasts, expression of MyoD decreased significantly compared to TRF-treated young myoblasts $(p<0.05)$. 

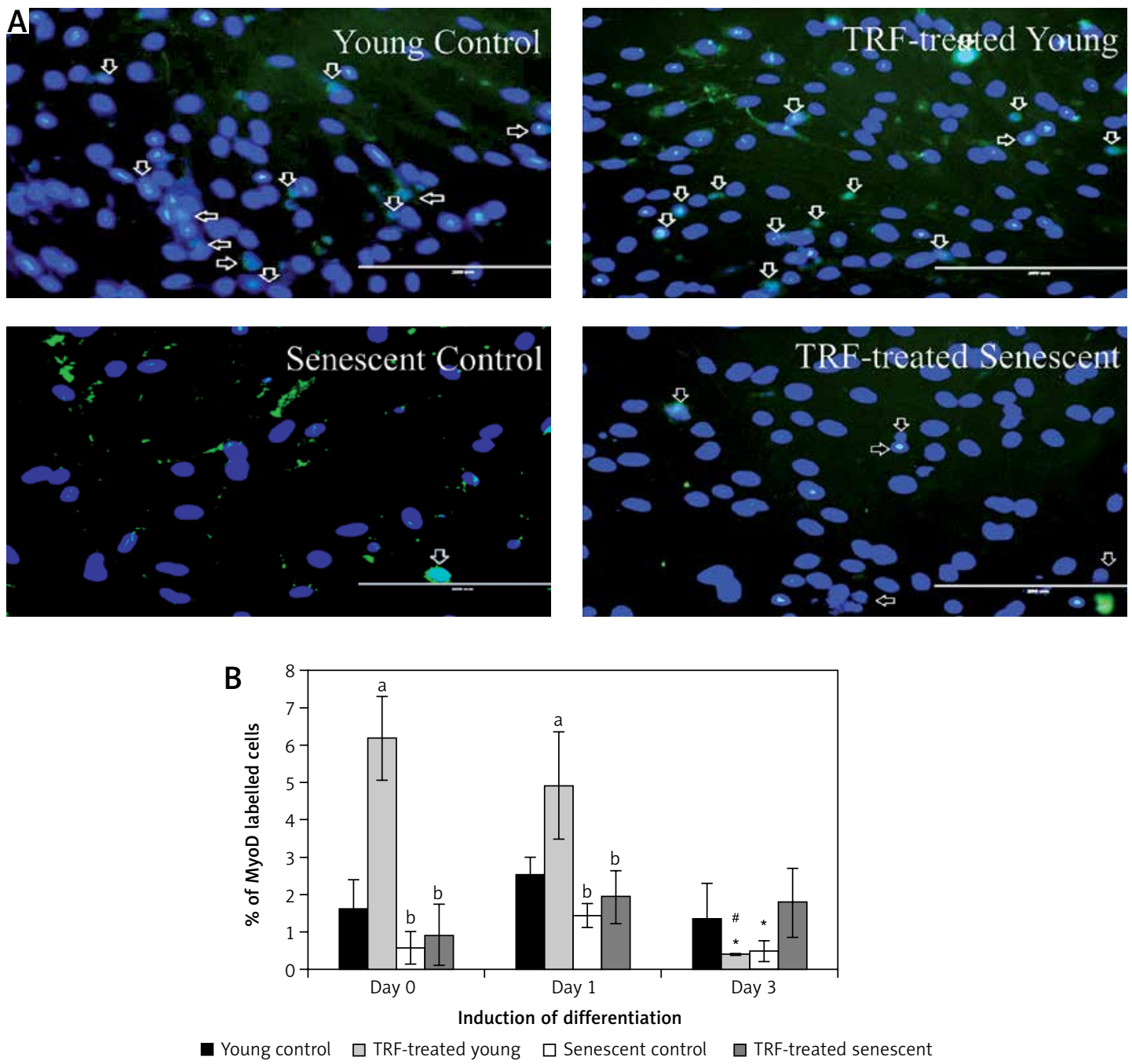

Figure 3. Effects of replicative senescence and TRF treatment on the expression of MyoD. A - Myoblasts were labelled with anti-MyoD antibody (green) and Hoechst (blue) to detect the presence of MyoD protein, as indicated by white arrow (magnification 200x, scale bar: $200 \mu \mathrm{m}$ ). B - Quantitative analysis of MyoD expression in myoblasts at day 1 , day 3 and day 5 of differentiation

${ }^{a}$ Denotes $p<0.05$, significantly different compared to young control at corresponding day of differentiation; ${ }^{b} p<0.05$, significantly different compared to TRF-treated young myoblasts at corresponding day of differentiation; $c p<0.05$, significantly different compared to senescent control at corresponding day of differentiation; ${ }^{*} p<0.05$, significantly different compared to the corresponding group at day 1 of differentiation; and ${ }^{*} p<0.05$, significantly different compared to the corresponding group at the day 3 of differentiation. Data are presented as the mean $\pm S D, n=3$.

Myogenin expression was progressively increased after differentiation induction, peaked at day 3 of differentiation, and then decreased at day 5 of differentiation in young control myoblasts ( $p<0.05$; Figure $4 \mathrm{~B}$ ). However, the myogenin expression pattern of the senescent control was different, showing a significantly lower level of myogenin from day 1 until day 5 of differentiation compared to young control myoblasts $(p<0.05)$. Treatment with TRF increased myogenin expression in senescent myoblasts compared to the untreated senescent control as early as day 1 until day 5 of differentiation. There was a progressive increase in myogenin expression in TRF-treated young and senescent myoblasts until day 3 of differentiation which was maintained at day 5 of dif- ferentiation ( $p<0.05$; Figure 4 B). Treatment with TRF also caused a significant increase in myogenin expression in young myoblasts at day 5 of differentiation compared to young control myoblasts $(p<0.05)$.

\section{Improvement in efficiency of myogenic differentiation with TRF treatment}

To evaluate the beneficial effects of TRF on the efficiency of myogenic differentiation, myoblasts were permitted to differentiate for 5 days to form myotubes and the morphology of myotubes was observed, while the fusion index and size of myotubes were measured (Figure 5). The myotubes 

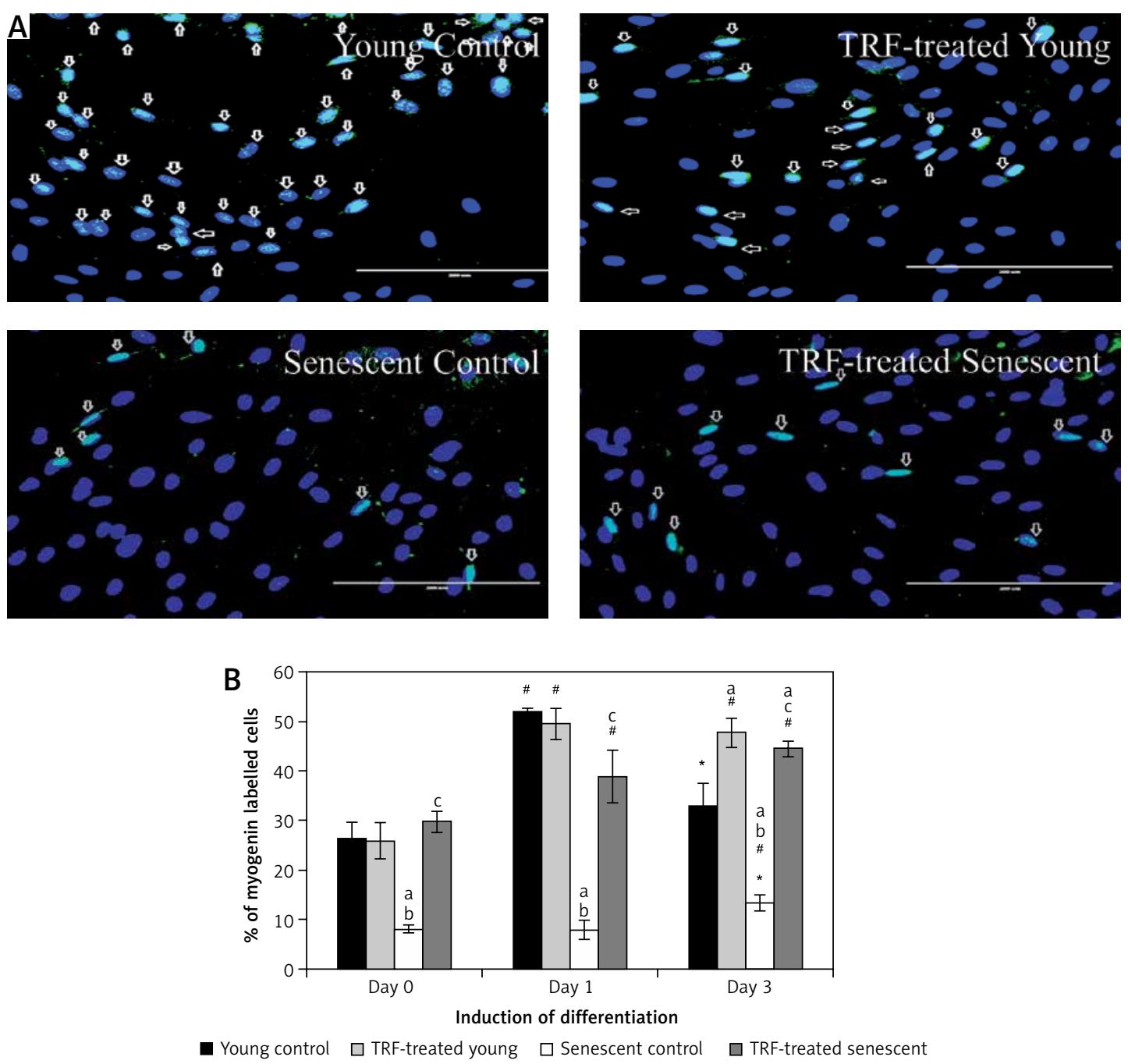

Figure 4. Effects of replicative senescence and TRF treatment on the expression of myogenin. A - Myoblasts were labelled with anti-Myogenin antibody (green) and Hoechst (blue) to detect the presence of myogenin protein, as indicated by white arrow (magnification 200x, scale bar: $200 \mu \mathrm{m}$ ). B - Quantitative analysis of myogenin expression in myoblasts at day 1 , day 3 and day 5 of differentiation

${ }^{a}$ Denotes $p<0.05$, significantly different compared to young control at corresponding day of differentiation; ${ }^{b} p<0.05$, significantly different compared to TRF-treated young myoblasts at corresponding day of differentiation; ${ }^{*}<0.05$, significantly different compared to the corresponding group at day 1 of differentiation; and ${ }^{*} p<0.05$, significantly different compared to the corresponding group at the day 3 of differentiation. Data are presented as the mean $\pm S D, n=3$.

formed by young myoblasts were large and multinucleated (Figure $5 \mathrm{~A}$ ). In contrast, senescent cells formed smaller myotubes with fewer nuclei compared to young cells (Figure $5 \mathrm{~A}$ ), indicating an incompetent differentiation process during the replicative senescence of myoblasts. A steady increase of the fusion index was observed in both young and senescent cells throughout the differentiation induction, but the fusion index of senescent cells at day 1 , day 3 and day 5 of differentiation was significantly lower compared to the young control at the corresponding day of differentiation $(p<0.05$; Figure 5 B). Moreover, a similar steadily increase of myotubes size was observed in young control myoblasts throughout the differentiation induction as indicated by the elevated number of nuclei per myotube ( $p<0.05$; Figure $5 \mathrm{C})$. The senescent control exhibited significantly larger myotubes size only at day 5 of differentiation compared to myotube size at day 3 of differentiation $(p<0.05)$. The size of myotubes of senescent cells however was smaller as compared to the young control at day 3 and day 5 of differentiation $(p<0.05)$.

Treatment with TRF improved the differentiation of senescent myoblasts to multinucleated myotubes, even though they were still smaller in size compared to young myoblasts (Figure $5 \mathrm{~A}$ ). Quantitative analysis showed that TRF significantly increased the fusion index and the size of myotubes of senescent cells compared to the untreated control $(p<0.05)$ (Figures 5 B, C). Although TRF-treated senescent cells had a higher fusion in- 

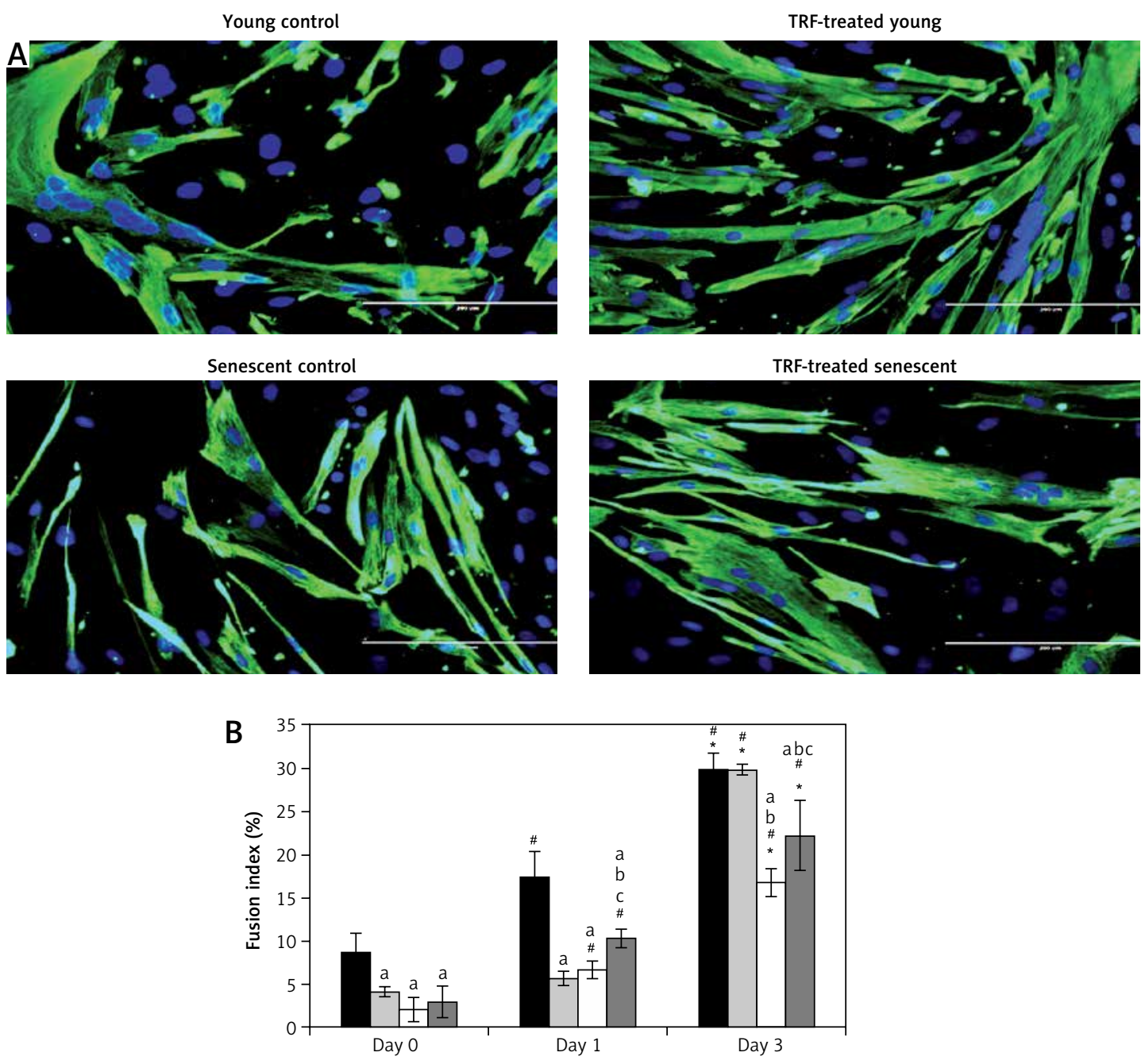

Induction of differentiation

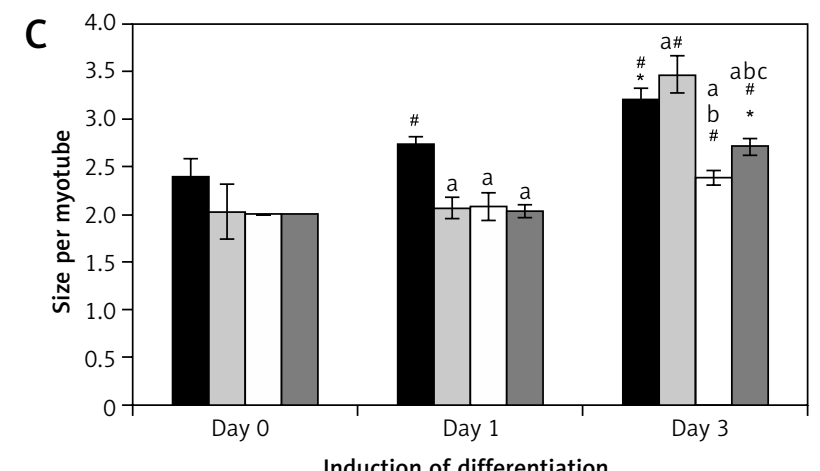

- Young control $\square$ TRF-treated young $\square$ Senescent control $\square$ TRF-treated senescent

Figure 5. Effects of replicative senescence and TRF treatment on the efficiency of myogenic differentiation. A - Micrographs showed the formation of myotubes in each group (magnification 200x, scale bar: $200 \mu \mathrm{m}$ ). Multinucleated myotubes were observed in young groups, conversely myogenic differentiation was impaired during replicative senescence. B - The fusion index and C - the size of myotubes were determined, showing that TRF was able to increase the differentiation potential of senescent myoblasts

${ }^{a} p<0.05$, significantly different compared to young control at corresponding day of differentiation; ${ }^{b} p<0.05$, significantly different compared to TRF-treated young myoblasts at corresponding day of differentiation; $c p<0.05$, significantly different compared to senescent control at corresponding day of differentiation; ${ }^{*} p<0.05$, significantly different compared to the corresponding group at day 1 of differentiation; and ${ }^{*} p<0.05$, significantly different compared to the corresponding group at the day 3 of differentiation. Data are presented as the mean $\pm S D, n=3$. 
dex at day 3 and day 5 of differentiation compared to the senescent control at the corresponding day of differentiation $(p<0.05)$, the levels were still significantly lower compared to the young control $(p<0.05$; Figure 5 B). A similar pattern was observed in the size of myotubes, in which TRF-treated senescent cells formed larger myotubes compared to the senescent control at day 5 of differentiation. However, the size of these myotubes was significantly smaller compared to the myotubes formed in the young control ( $p<0.05$; Figure $5 \mathrm{C}$ ).

\section{Discussion}

The efficiency of skeletal muscle regeneration relies on the regenerative potential of satellite cells, which may undergo senescence, and attenuate its regenerative capacity with aging [11]. Serial passaging provides a way to produce aged myoblasts in vitro. This extensive cell expansion exhausts the proliferative capacity of myoblasts, which leads to replicative senescence. Young myoblasts exhibit thin and spindle shape morphology. Meanwhile, senescent myoblasts are characterized by broader and flatter cytoplasm with an abundance of intermediate filaments. The phenotype changes observed in senescent cells resemble alterations at the molecular level. Senescent myoblasts showed a high level of ROS production, low proliferative capacity, downregulation of myogenic differentiation genes and high expression of oxidative damage-associated genes $[15,28]$. Our previous study showed that treatment with TRF in senescent myoblasts resulted in regained morphology of young cells indicated by the presence of more spindle-shaped cells, suggesting that TRF could be used as a potential compound for reversing muscle aging [15].

Tocotrienol-rich fraction has been shown to have not only free radical scavenging power but could also reduce the senescent phenotype of the stress-induced premature senescence (SIPS) model of myoblasts [27]. Its effects were also superior to $\alpha$-tocopherol in promoting myogenic differentiation of senescent myoblasts [15]. Emerging data indicates that tocotrienols are promising therapeutic agents which could prevent cancer, diabetes, and cardiovascular and neurodegenerative diseases [29]. Additionally, TRF may confer protection against oxidative stress-induced injury in rat skeletal muscle and preserves the well-functioning muscle performance [30]. Increased proliferation capacity and modulation of MRF expression by TRF also reveals its potential in restoring the impaired regenerative capacity of the muscle during aging [15]. In this study, we further illustrated the potential of TRF in improving myoblast proliferation and differentiation by modulating the proliferation- and differentiation-associated protein expression in senescent human myoblasts during the differentiation phase.

Desmin expression varies in myoblasts derived from different donors. Some of them are able to maintain the expression of desmin after extensive expansion and others are not [31]. In this study, senescent myoblasts lost their desmin content, which might be associated with declined proliferation capacity at the point of senescence, resulting in fibroblasts being outgrown with reduced myogenic purity [32]. In spite of that, no significant changes concerning myogenic purity were observed with TRF treatment, which is in agreement with previous findings [27] and allows comparison between TRF-treated cells and the untreated control.

Ki67, a nuclear protein of mammalian cells, is closely associated with somatic cell proliferation. Ki67 protein is present in all proliferating cells in cluding normal and tumor cells that have the capacity to proliferate. In addition, this protein is $a b$ sent in quiescent cells or cells that are arrested at $\mathrm{G}_{0}$ phase, both types of cells having lost the proliferative capacity [33]. This protein has been widely used as a marker of cell proliferation, especially to monitor cellular proliferation in human tumors [34-36]. Furthermore, expression of Ki67 is also used in estimating the percentage of active satellite cells (myoblasts) [37]. Normally, myoblasts undergo active proliferation before entering the differentiation phase, which involves cell cycle arrest and fusion into myotubes [38]. However, the level of Ki67 expression in senescent myoblasts was relatively low compared to young myoblasts, which was similar to the expression in aged mus cle [39]. A previous study also reported that there was a decline in cellular proliferation in senescent myoblasts compared to young myoblasts [15]. After induction of differentiation, the Ki67 expression in myoblasts was gradually decreased [32]. In this study, instead of expressing an extremely low level of Ki67 protein from day 3 of differentiation as observed in senescent control, TRF-treated senescent myoblasts showed elevated expression of Ki67 to the extent that it was similar to young myoblasts, indicating the potential of TRF in in creasing the proliferation capacity of aging myoblasts, even during differentiation induction.

Our study also elucidated the role of MyoD during cell proliferation and differentiation. MyoD is a protein that links to the blockade of cell cycle progression when myogenic differentiation is induced [38]. Increased activity of MyoD at the onset of differentiation has been reported [16]. The MyoD expression in our study remained unchanged in all groups from day 1 to day 3 of differentiation, and decreased at day 5 of differentiation in TRF-treated young cells and the senescent control. For TRF-treated young cells, our findings 
are in conjunction with MYOD1 mRNA expression, which was up-regulated at day 1 and day 3 of differentiation compared to the untreated control [15]. However, in this study TRF did not increase MyoD expression in senescent myoblasts as has been observed on its mRNA expression [15], suggesting that MyoD protein expression did not correlate completely with its gene expression. MyoD has an important regulatory role in initiating myogenic differentiation [38, 40]. Defects in differentiation and at the proliferation-differentiation transition were observed in satellite cells derived from adult $\mathrm{MyoD}^{-/-}$mice [40]. Thus, increased MyoD expression in young myoblasts with TRF treatment at day 1 and day 3 of differentiation induction observed in this study revealed the effectiveness of TRF in promoting the differentiation process in myoblasts.

Myogenin expression is controlled by MyoD, in which the up-regulation of myogenin occurs subsequent to the MyoD in young myoblasts $[16,41]$. However, the regulatory role of myogenin in retaining myogenic differentiation cannot be compensated by other MRFs [42]. Our results showed that myogenin expression was increased during differentiation, but the increase was markedly lower in senescent myoblasts, where it was significantly increased only at day 5 of differentiation induction. Findings from previous studies reported a similar setback in both gene and protein expression of myogenin in senescent myoblasts, indicating impaired myogenic differentiation regulation during replicative senescence [16, 32]. Delayed myogenin protein expression was also observed in old-individual-derived muscle [18]. In this study, we found that TRF improved the expression of myogenin in senescent myoblasts from day 1 of differentiation, which is similar to our previous findings which showed that TRF can ameliorate muscle differentiation by increasing the expression of myogenin at both mRNA and protein levels [15]. Hence, treatment with TRF could be a relevant strategy to modulate myogenin expression for promoting myogenic differentiation.

Previous studies reported that senescent myoblasts are unlikely to differentiate and fuse to form multinucleated myotubes in comparison to young myoblasts, in which young myoblasts are able to form large and branched myotubes upon differentiation induction [6, 14-16]. This is in agreement with our current results which demonstrated that the formation of myotubes was impaired by replicative senescence, hence resulting in a lower fusion index and a smaller size of myotubes compared to young myoblasts. Moreover, the declined efficacy of differentiation in senescent myoblasts is in conjunction with decreased myogenin expression observed in this study.

TRF is a broad mixture of vitamin $E$ that contains all four isomers of tocotrienol and $\alpha$-tocopherol, which has been found to be beneficial for promotion of myogenic differentiation during replicative senescence [15]. Based on the morphology observation and quantification of fusion index and size of myotubes, our results showed that TRF treatment effectively ameliorates the defective differentiation program in senescent myoblasts, as manifested by the improvement of myotube formation. However, TRF treatment did not give a positive effect on the differentiation of young myoblasts. A previous study on humans showed that supplementation of TRF to a young group did not have any significant effect on lipid profile and oxidative status. In contrast, a significant result was observed in older subjects [43]. The difference in results observed between young and older subjects may reflect the fact that the biological system in young cells is well-maintained particularly at the redox balance and oxidative damage level. Furthermore, as the bioavailability of vitamin $E$ represents its biological activity, the homeostasis of this vitamin in the cell is tightly regulated to prevent toxicity and alteration to the biological system. A previous study showed that the uptake of TRF in senescent cells was higher than in young cells [44]. A similar finding was observed in elderly people, which showed higher uptake of TRF as compared to the young age group [43]. As the cells senesce, the oxidant level is proportionately increased, resulting in higher numbers of oxidized molecules which subsequently altered the biological system. Therefore, the effect of TRF was more intense and efficient on senescent cells compared to young cells.

In conclusion, the present study supports the potential of TRF treatment in modulating muscle regeneration during aging. Our results have demonstrated that TRF possessed a remarkable potential in promoting muscle regeneration by up-regulating the expression of proliferation (Ki67) and differentiation (myogenin) markers. TRF may be used as a therapeutic agent for ameliorating the differentiation defects in aged or sarcopenic muscle. However, further research should be carried out on other myoblast differentiation markers which fall under MRFs or muscle-related proteins such as MHC and MRF4.

\section{Acknowledgments}

This study was financially supported by Universiti Kebangsaan Malaysia Grant UKM-FF-2015-144 and a Ministry of Higher Education Grant (FRGS/2/2014/ SKK01/UKM/01/1). 


\section{Conflict of interest}

The authors declare no conflict of interest.

\section{References}

1. Carosio S, Berardinelli MG, Aucello M, Musaro A. Impact of ageing on muscle cell regeneration. Ageing Res Rev 2011; 10: 35-42.

2. Mauro A. Satellite cell of skeletal muscle fibers. J Biophys Biochem Cytol 1961; 9: 493.

3. Fu X, Wang H, Hu P. Stem cell activation in skeletal muscle regeneration. Cell Mol Life Sci 2015; 72: 1663-77.

4. Yin H, Price F, Rudnicki MA. Satellite cells and the muscle stem cell niche. Physiol Rev 2013; 93: 23-67.

5. Blau HM, Cosgrove BD, Ho AT. The central role of muscle stem cells in regenerative failure with aging. Nat Med 2015; 21: 854-62

6. Barberi L, Scicchitano BM, De Rossi M, et al. Age-dependent alteration in muscle regeneration: the critical role of tissue niche. Biogerontology 2013; 14: 273-92.

7. Brack AS, Rando TA. Intrinsic changes and extrinsic influences of myogenic stem cell function during aging. Stem Cell Rev 2007; 3: 226-37.

8. Conboy IM, Conboy MJ, Wagers AJ, Girma ER, Weissman IL, Rando TA. Rejuvenation of aged progenitor cells by exposure to a young systemic environment. Nature 2005; 433: 760-4.

9. Conboy IM, Rando TA. Heterochronic parabiosis for the study of the effects of aging on stem cells and their niches. Cell Cycle 2012; 11: 2260-7.

10. Zammit P, Heslop L, Hudon V, et al. Kinetics of myoblast proliferation show that resident satellite cells are competent to fully regenerate skeletal muscle fibers. Exp Cell Res 2002; 281: 39-49.

11. Sousa-Victor P, Gutarra S, Garcia-Prat L, et al. Geriatric muscle stem cells switch reversible quiescence into senescence. Nature 2014; 506: 316-21.

12. Mouly V, Aamiri A, Bigot A, et al. The mitotic clock in skeletal muscle regeneration, disease and cell mediated gene therapy. Acta Physiol Scand 2005; 184: 3-15.

13. Yang R, Fang W, Liang J, et al. Apelin/APJ axis improves angiotensin II-induced endothelial cell senescence through AMPK/SIRT1 signaling pathway. Arch Med Sci 2018; 14: 725-34.

14. Lorenzon P, Bandi E, de Guarrini F, et al. Ageing affects the differentiation potential of human myoblasts. Exp Gerontol 2004; 39: 1545-54.

15. Khor SC, Razak AM, Wan Ngah WZ, Mohd Yusof YA, Abdul Karim N, Makpol S. The tocotrienol-rich fraction is superior to tocopherol in promoting myogenic differentiation in the prevention of replicative senescence of myoblasts. PLoS One 2016; 11: e0149265.

16. Bigot A, Jacquemin V, Debacq-Chainiaux F, et al. Replicative aging down-regulates the myogenic regulatory factors in human myoblasts. Biol Cell 2008; 100: 189-99.

17. Weintraub H, Davis R, Tapscott S, et al. The MyoD gene family: nodal point during specification of the muscle cell lineage. Science 1991; 251: 761-6.

18. Alway SE, Lowe DA, Chen KD. The effects of age and hindlimb supension on the levels of expression of the myogenic regulatory factors MyoD and myogenin in rat fast and slow skeletal muscles. Exp Physiol 2001; 86 509-17.

19. Vasilaki A, Jackson MJ. Role of reactive oxygen species in the defective regeneration seen in aging muscle. Free Radic Biol Med 2013; 65: 317-23.
20. Mocchegiani E, Costarelli L, Giacconi R, et al. Vitamin E-gene interactions in aging and inflammatory age-related diseases: implications for treatment. A systematic review. Ageing Res Rev 2014; 14: 81-101.

21. Rafique R, Schapira AH, Cooper JM. Mitochondrial respiratory chain dysfunction in ageing; influence of vitamin E deficiency. Free Radic Res 2004; 38: 157-65.

22. Nier B, Weinberg PD, Rimbach G, Stocklin E, Barella L. Differential gene expression in skeletal muscle of rats with vitamin E deficiency. IUBMB Life 2006; 58: 540-8.

23. Sacheck JM, Milbury PE, Cannon JG, Roubenoff R, Blumberg JB. Effect of vitamin $E$ and eccentric exercise on selected biomarkers of oxidative stress in young and elderly men. Free Radic Biol Med 2003; 34: 1575-88.

24. Howard AC, McNeil AK, McNeil PL. Promotion of plasma membrane repair by vitamin E. Nat Commun 2011; 2: 597.

25. Sen CK, Khanna S, Roy S, Tocotrienols: vitamin E beyond tocopherols. Life Sci 2006; 78: 2088-98.

26. Ali SF, Woodman OL. Tocotrienol rich palm oil extract is more effective than pure tocotrienols at improving endothelium-dependent relaxation in the presence of oxidative stress. Oxid Med Cell Longev 2015; 2015: 150829.

27. Lim JJ, Ngah WZ, Mouly V, Abdul Karim N. Reversal of myoblast aging by tocotrienol rich fraction posttreatment. Oxid Med Cell Longev 2013; 2013: 978101.

28. Pääsuke R, Eimre M, Piirsoo A, et al. Proliferation of human primary myoblasts is associated with altered energy metabolism in dependence on ageing in vivo and in vitro. Oxid Med Cell Longev 2016; 2016: 8296150.

29. Aggarwal BB, Sundaram C, Prasad S, Kannappan R. Tocotrienols, the vitamin E of the $21^{\text {st }}$ century: its potential against cancer and other chronic diseases. Biochem Pharmacol 2010; 80: 1613-31.

30. Lee SP, Mar GY, Ng LT. Effects of tocotrienol-rich fraction on exercise endurance capacity and oxidative stress in forced swimming rats. Eur J Appl Physiol 2009; 107: 587-95.

31. Schäfer R, Knauf U, Zweyer M, et al. Age dependence of the human skeletal muscle stem cell in forming muscle tissue. Artif Organs 2006; 30: 130-40.

32. Alsharidah M, Lazarus NR, George TE, Agley CC, Velloso CP, Harridge SD. Primary human muscle precursor cells obtained from young and old donors produce similar proliferative, differentiation and senescent profiles in culture. Aging Cell 2013; 12: 333-44.

33. Scholzen T, Gerdes J. The Ki-67 protein: from the known and the unknown. J Cell Physiol 2000; 182: 311-22.

34. Endl E, Gerdes J. The Ki-67 protein: fascinating forms and an unknown function. Exp Cell Res 2000; 257: 231-7.

35. Bruey JM, Kantarjian H, Ma W, et al. Circulating Ki-67 index in plasma as a biomarker and prognostic indicator in chronic lymphocytic leukemia. Leuk Res 2010; 34: 1320-4.

36. Gurzu S, Orlowska J, Sugimura H, et al. Immunohistochemical features and staging of early gastric cancer. Arch Med Sci 2017; 13: 1373-82.

37. Mackey AL, Kjaer M, Charifi N, et al. Assessment of satellite cell number and activity status in human skeletal muscle biopsies. Muscle Nerve 2009; 40: 455-65.

38. Kitzmann M, Fernandez A. Crosstalk between cell cycle regulators and the myogenic factor MyoD in skeletal myoblasts. Cell Mol Life Sci 2001; 58: 571-9.

39. Hu Z, Klein JD, Mitch WE, Zhang L, Martinez I, Wang XH. MicroRNA-29 induces cellular senescence in aging muscle through multiple signaling pathways. Aging (Albany NY) 2014; 6: 160-75. 
40. Yablonka-Reuveni Z, Rudnicki MA, Rivera AJ, Primig M, Anderson JE, Natanson P. The transition from proliferation to differentiation is delayed in satellite cells from mice lacking MyoD. Dev Biol 1999; 210: 440-55.

41. Cao Y, Yao Z, Sarkar D, et al. Genome-wide MyoD binding in skeletal muscle cells: a potential for broad cellular reprogramming. Dev Cell 2010; 18: 662-74.

42. Lee EJ, Malik A, Pokharel S, et al. Identification of genes differentially expressed in myogenin knock-down bovine muscle satellite cells during differentiation through RNA sequencing analysis. PLoS One 2014; 9: e92447

43. Chin SF, Ibahim J, Makpol S, et al. Tocotrienol rich fraction supplementation improved lipid profile and oxidative status in healthy older adults: a randomized controlled study. Nutr Metab (Lond) 2011; 8: 42.

44. Jaafar F, Abdullah A, Makpol S. Cellular uptake and bioavailability of tocotrienol-rich fraction in SIRT1-inhibited human diploid fibroblasts. Sci Rep 2018; 8: 10471. 